\title{
Les enjeux politiques de la mémoire du passé colonial à l'île Maurice
}

Dans une île peuplée de migrants de diverses origines et de statuts inégaux, une mémoire nationale n'émerge que difficilement

Political Issues of the Memory of the Colonial Past in Mauritius

\section{Catherine Boudet et Julie Peghini}

\section{CpenEdition}

\section{Journals}

Édition électronique

URL : http://journals.openedition.org/transcontinentales/397

DOI : $10.4000 /$ transcontinentales.397

ISBN : 978-2-7351-1561-7

ISSN : 1775-397X

Éditeur

Editions de la maison des sciences de l'homme

\section{Édition imprimée}

Date de publication : 30 juin 2008

Pagination : 13-36

ISBN : 978-2-200-92511-6

ISSN : 1950-1684

Référence électronique

Catherine Boudet et Julie Peghini, «Les enjeux politiques de la mémoire du passé colonial à l'île Maurice », Transcontinentales [En ligne], 6 | 2008, document 1, mis en ligne le 06 avril 2011, consulté le 08 septembre 2020. URL : http://journals.openedition.org/transcontinentales/397 ; DOI : https:// doi.org/10.4000/transcontinentales.397 


\title{
Les enjeux politiques de la mémoire du passé colonial à l'île Maurice
}

\author{
Catherine Boudet et Julie Peghini
}

\begin{abstract}
À l'île Maurice, en quarante ans d'indépendance, le modèle étatique d'«unité dans la diversité», fondé sur un pluralisme ethnique reconnu constitutionnellement ${ }^{1}$, ne s'est pas accompagné de l'émergence d'une mémoire collective, d'un récit fondateur de la collectivité nationale. La division de la société en termes communautaires, héritée de la période coloniale et maintenue dans l'État indépendant, a encouragé au contraire l'entretien de mémoires ethniques.
\end{abstract}

Cet éclatement des registres mémoriels a été historiquement déterminé par la constitution de la société mauricienne par vagues de migrations successives, correspondant aux phases de l'esclavage (aboli en 1835) et à l'arrivée des travailleurs indiens au titre de $l^{\prime}$ «engagisme ${ }^{2} »(1834-1907)$. Pour des groupes ethniques arrivés à des moments différents de l'histoire et s'insérant dans des rapports de pouvoir historiques du fait des systèmes d'exploitation coloniaux qu'ont constitué l'esclavage et l'engagisme, la détermination d'un "moment-origine» nécessaire à la fondation d'une communauté nationale ${ }^{3}$ ne pouvait revêtir la même signification. De ce fait, l'appropriation d'une mémoire collective est immanquablement aux prises avec le problème d'une concurrence sur les représentations du passé colonial.

1 - Les communautés ethniques, reconnues au nombre de quatre (Hindous 51,8 \%, Population Générale 28,7 \%, Musulmans 16,6 \% et Sino-Mauriciens 2,9 \%) par la Constitution de 1968, furent officiellement supprimées en 1972. Elles n'en restent pas moins le fondement du système politique, par le biais du système du best loser (ou «meilleur perdant»), mécanisme électoral correctif ayant vocation à désigner des députés pour représenter les groupes minoritaires lorsque le résultat des élections n'a pas permis cette représentation.

2 - Système de salariat contraint, en vertu duquel une main-d'œuvre bon marché a été importée de l'Inde sous contrat pour remplacer les esclaves affranchis sur les plantations sucrières au moment de l'abolition de l'esclavage.

3 - J. Candau, Mémoire et identité, Paris, Presses universitaires de France, 1998.

Catherine Boudet : Université de la Réunion, Institut français d'Afrique du Sud (IFAS), Johannesburg. Julie Peghini : Université Paris-VIII, IFAS à Johannesburg. 
On peut ainsi distinguer trois grands registres de la mémoire du passé colonial à Maurice, en rapport avec les identifications ethniques : la mémoire de la colonisation fondatrice, à laquelle s'identifient les Franco-Mauriciens et la partie la moins métissée de la Population Générale ${ }^{4}$; la mémoire de l'esclavage à laquelle s'identifient les Créoles et la partie la plus métissée de la Population Générale; et enfin la mémoire de l'engagisme à laquelle s'identifie la communauté hindoue ${ }^{5}$. Il convient de souligner à cet égard que les registres mémoriels et les appartenances communautaires ne coïncident pas de façon catégorique ${ }^{6}$.

L'émergence d'une mémoire collective à Maurice se situe ainsi à la croisée d'une triple problématique: l'éclatement des registres de mémoire ethnicisés, d'où l'absence d'une vision unifiée de l'histoire coloniale; des tiraillements entre les deux niveaux de production de la mémoire, ethnique et étatique; et enfin, l'accès inégalitaire des groupes ethniques à la mémoire.

Nous verrons comment les Franco-Mauriciens, dans un souci d'inclusion dans la nation, procèdent à une édulcoration du passé colonial pour le dépouiller de ses attributs de domination (section 1); tandis que les élites hindoues, disposant de l'appareil de l'État, tentent de réhabiliter la mémoire des lésés de l'histoire mauricienne, les engagés et les esclaves, afin d'asseoir leur pouvoir politique sur le registre symbolique que leur fournit cette mémoire (section 2). Les Créoles, laissés pour compte dans le développement de la nation indépendante, tentent de se réapproprier la mémoire de l'esclavage en élaborant une contre-histoire pour se forger une mémoire ethnique et ainsi acquérir des ressources symboliques dans la compétition sur les ressources de l'État (section 3). Dès lors, les usages politiques du passé colonial dans l'espace public permettent-ils d'effectuer des ajustements réciproques au sein du modèle national, dans le sens de l'émergence d'une mémoire nationale unifiée, ou favorisent-ils au contraire un regain de compétition interethnique? (section 4)

4 - Cette catégorie institutionnelle réunit les groupes descendant de la société esclavagiste, suivant une hiérarchie pigmentaire héritée de cette période : les Franco-Mauriciens (autrefois les «Blancs»), les gens de "la frontière", les "Gens de couleur» (autrefois "Libres de couleur») et les "Créoles" (autrefois les esclaves). Au fil du temps, elle s'est également augmentée de tous les cas de métissage et de conversion aux religions chrétiennes (Tamouls baptisés, Chinois baptisés), en vertu desquels les individus concernés n'étaient plus identifiables aux trois autres communautés.

5 - La communauté hindoue englobe également des minorités, qui partagent la même religion avec des variantes héritées de leur provenance régionale en Inde: les Tamouls, les Télougous, les Marathis. La comunauté hindoue majoritaire est, elle, principalement issue du Bihar.

6 - Ainsi les Musulmans, pourtant originaires de l'Inde, ne s'identifient pas nécessairement à la mémoire de l'engagisme, dans la mesure où, d'une part, leurs ancêtres ont fait partie de l'immigration libre et où, d'autre part, la tendance actuelle est à l'identification sur un registre religieux à l'Ummah en association ou en concurrence avec une identification à l'Inde sur un registre ethnique. Quant aux Sino-Mauriciens, ils sont à la croisée des deux registres de mémoire, ayant mis en place une démarche mémorielle pour connaître le rôle de l'esclavage et celui de l'engagisme dans l'immigration chinoise à Maurice. 
Les Franco-Mauriciens

et l'édulcoration de la mémoire coloniale

Historiquement, le premier groupe dans lîle à produire la mémoire d'un «momentorigine» est celui des Franco-Mauriciens, ou Mauriciens blancs d'origine française. Le «moment-origine» joue un rôle fondateur de l'identité ethnique, dans la mesure où il a vocation à "naturaliser la communauté», à proclamer son identité et à la pourvoir d'une cohésion ${ }^{7}$. Il est le levier par lequel se constitue pendant la colonisation britannique (1810-1968) un groupe franco-mauricien à partir de ce qui était antérieurement le groupe des colons blancs d'origine française.

Cette minorité dominante issue de la colonisation de l'île par la France par droit de conquête perd sous la colonisation britannique les fondements structurels de sa domination, à savoir la «barrière de couleur» de la société esclavagiste et le pouvoir politique. Elle résiste cependant aux décadences successives de sa puissance par l'acquisition du monopole sucrier (1820) et par l'investissement du pouvoir législatif, ce qui lui permet de se constituer en une "oligarchie sucrière» et d'entrer dans un rapport d'association et de lobbying avec l'administration coloniale britannique ${ }^{8}$.

La référence à l'antériorité sur le sol de lîle devient alors un argument culturel de légitimation de la domination minoritaire. La conquête et la colonisation de l'île par la France acquièrent pour la minorité franco-mauricienne la dimension d'une «mythohistoire», c'est-à-dire d'une histoire ayant la fonction ontologique du mythe d'origine. Cette mytho-histoire a vocation de modèle explicatif à la fois de la fondation de lîle et du système social hiérocratique ${ }^{9}$. Le «moment-origine» de la période française fournit au groupe l'argument culturel qui légitime sa position dominante en l'asseyant sur la qualité de primo-arrivant fondateur de la société insulaire, et sa lutte pour la préservation de droits spécifiques vis-à-vis de l'administration britannique ${ }^{10}$.

Avec la décolonisation (1947-1968), l'oligarchie sucrière perd le pouvoir politique formel et se voit contrainte de composer avec les autres élites ethniques au sein $\mathrm{d}^{\prime}$ un système consociatif ${ }^{11}$. La référence à la mytho-histoire de la période française soutient alors des stratégies d'alliances électorales avec les élites du groupe de couleur tout en permettant d'invoquer le clivage avec la majorité hindoue : «les forêts ne recelaient point d'autochtones. Personne ne peut en conséquence revendiquer des droits

7 - J. Candau, Mémoire et identité, op. cit., p. 87.

8 - C. Boudet, "Pouvoirs et technologies en situation coloniale: les Franco-Mauriciens et le monopole des technologies sucrières à Maurice et au Natal (1825-1968)", Revue historique de l'océan Indien, $\mathrm{n}^{\circ}$ 2, AHIOI, 2006, p. 178-193.

9 - C'est-à-dire qui s'organise de façon coercitive en une pyramide hiérarchisant des groupes économiquement complémentaires mais considérés comme socialement, culturellement, voire racialement inégaux, sous la domination d'une minorité européenne. R.A. Schermerhorn, Comparative Ethnic Relations, A Framework for Theory and Research, New York, Random House, 1968.

10 - C. Boudet, «Les Franco-mauriciens : une identité pollinisée», Revue européenne des migrations internationales, vol. 23 (3), 2007, p. 109-131.

11 - La consociation désigne un système politique d'évitement des conflits et de partage du pouvoir entre élites représentant les différents groupes ethniques en présence, en situation d'interdépendance contrainte. 
antérieurs à ceux des Européens. [...] La fondation de la colonie fut l'œuvre exclusive des colons français assistés de leurs esclaves d'origine africaine ${ }^{12}$ ", écrit en 1954 Noël Marrier d'Unienville, rédacteur en chef du Cernéen, le journal de l'élite sucrière, pour affirmer en contrepoint que "les Indiens sont complètement étrangers à la fondation de l'île et [...] ont ensuite formé une main-d'œuvre utile certes et méritoire mais non organisatrice» (Le Cernéen du 7 novembre 1955). Cette mise en opposition a vocation à délégitimer la revendication des élites hindoues en faveur de l'indépendance. Ce faisant, l'usage politique de la mytho-histoire comme volet discursif des stratégies électorales signale l'émergence d'une catégorie de discours fondé sur la contribution à la fondation de l'île comme argument de légitimité politique.

L'indépendance consacre une rupture dans le rapport du groupe franco-mauricien à sa mémoire, par la recherche d'élision au plan discursif de son caractère de dominant. En effet, les élites franco-mauriciennes conservant le monopole sucrier, pilier de l'économie mauricienne, l'enjeu est désormais de préserver la relation de partage du pouvoir, inhérente au système consociatif, qui s'est instaurée avec la bourgeoisie hindoue nouvellement détentrice du pouvoir politique. Le souci de légitimer la place de la minorité (anciennement dominante) franco-mauricienne dans la nation indépendante se traduit par une "édulcoration» de la mémoire coloniale ${ }^{13}$ visant à gommer toute connotation hégémonique. La fermeture du journal franco-mauricien Le Cernéen ${ }^{14}$ atteste de cette démarche. En 1981, les chefs d'entreprise franco-mauriciens qui financent le quotidien demandent à son rédacteur en chef, Jean-Pierre Lenoir, de supprimer la référence à Adrien d'Épinay, le fondateur, en raison de son image d'esclavagiste. Le refus de Lenoir marque la fin du journal que ses financeurs ne considéraient déjà plus rentable, préférant désormais utiliser la presse générale pour leur communication.

L'enjeu n'est toutefois pas d'effacer la mémoire coloniale, fondatrice de l'ethnogenèse du groupe, mais de repositionner symboliquement celui-ci au sein de la nation indépendante. Le slogan de l'«unité dans la diversité», d'inspiration indienne, traduit en effet un modèle national où la légitimité de chaque groupe ethnique à accéder aux ressources nationales (le "gâteau national») est mesurée à l'aune de sa contribution à la démographie, à l'économie, voire même à l'histoire du pays. À cet égard, il fonctionne suivant le modèle du «bien limité» de Foster ${ }^{15}$, les groupes en présence agissant comme

12 - N. Marrier d'Unienville, L'île menacée, Port-Louis (Maurice), The General Printing and Stationary, 1954, p. 3.

13 - Le souci de présenter le système esclavagiste mauricien comme relativement clément (notamment par rapport aux Antilles) remonte au combat anti-abolitionniste entre 1823 et 1834, lorsque les planteurs franco-mauriciens eurent à s'organiser contre les attaques de l'Anti-Slavery Society, société philosophique londonienne représentée au Parlement britannique, qui proposait Maurice comme terrain d'essai pour une abolition sans indemnisation des propriétaires. C. Boudet, Les FrancoMauriciens entre Maurice et l'Afrique du Sud : identité, stratégies migratoires et processus de recommunautarisation, thèse de science politique, Sciences Po Bordeaux, 2004, p. 145.

14 - Le Cernéen, le premier journal de l'île, a été fondé en 1832 par des planteurs francomauriciens et leur chef de file, Adrien d'Épinay, le plus gros propriétaire d'esclaves de l'île, pour s'organiser contre l'abolition de l'esclavage et revendiquer le rétablissement des droits politiques hérités de la Révolution française qui avaient été abolis par l'autorité britannique.

15 - G. Foster, "Peasant Society and the Image of the Limited Good», American Anthropologist, $\mathrm{n}^{\circ} 4,1967$, p. 293-315. 
si les ressources étatiques disponibles l'étaient en quantité limitée et qu'en conséquence, toute ressource obtenue par une communauté le serait au détriment d'une autre, laquelle se sentirait alors spoliée $e^{16}$. La mytho-histoire de la période française continue de posséder pour le groupe franco-mauricien la fonction de justification/légitimation de sa contribution et partant, de sa position économique, face aux suspicions selon lesquelles il disposerait de plus de ressources économiques qu'il ne serait légitime ${ }^{17}$.

Dès lors, le groupe franco-mauricien se retrouve pris entre le souci de continuer de se référer à la mémoire de la "colonisation-fondation» et celui d'affirmer sa place dans la nation mauricienne, tension qu'il résout en édulcorant le passé colonial de façon à soutenir sa recherche de légitimité. Dans cette démarche, les musées privés tiennent une place importante et il n'est pas fortuit qu'ils soient l'initiative d'élites économiques franco-mauriciennes, liées aux intérêts sucriers, comme en témoignent le Blue Penny Museum et le musée de l'Aventure du sucre.

Le Blue Penny Museum est un musée privé d'histoire et d'art qui a été ouvert en 2001 grâce au mécénat de la Mauritius Commercial Bank ${ }^{18}$ afin de mettre en valeur la très riche collection de la banque, qui a débuté en 1953 avec l'acquisition à Londres des cartes originales de la prise de l'île par les Anglais. Le Blue Penny Museum valorise une collection d'objets d'art (notamment la statue de Paul et Virginie par le sculpteur franco-mauricien Prosper d'Épinay), mais aussi de numismatique, de philatélie ${ }^{19}$ et de cartographie, ainsi que des documents historiques sur l'histoire postale, maritime et aérienne, et sur la fondation de Port-Louis par Mahé de La Bourdonnais.

La localisation même du musée témoigne d'un processus d'édulcoration du passé colonial. Le Blue Penny Museum est situé sur la partie privée du front de mer de PortLouis, le Caudan Waterfront, construit en 1996 avec le financement de la MCB. À cet endroit, étaient installés autrefois les docks et le terminal du chemin de $\mathrm{fer}^{20}$. Les seules traces historiques sont les pierres des anciens bâtiments coloniaux (principalement

16 - Voir H. Hoetink, «Resource Competition, Monopoly and Racial Diversity», in L. A. Despres (éd.), Ethnicity and Resource Competition in Plural Societies, La Haye, Mouton Publishers, 1975, p. 9-21.

17 - En 1994, l'économiste Rajandra Paratian écrivait : «Si nous admettons que les usiniers-planteurs en tant qu'un groupe à part entière et les planteurs-propriétaires cultivant plus de 50 hectares forment partie de la grande propriété mauricienne, nous constatons que celle-ci représente 0,21 \% et contrôle 61,9 \% de la superficie plantée de cannes. Cette spécificité décerne à l'île Maurice le record mondial de la plus grande concentration de propriété foncière aux mains d'un groupuscule terrien ». R. Paratian, $L a$ république de l'île Maurice : dans le sillage de la délocalisation, Paris, L'Harmattan, 1994, p. 40-41.

18 - La MCB est la plus ancienne banque privée encore existante dans l'île. Fondée en 1833 avec des capitaux britanniques et franco-mauriciens pour financer la culture sucrière, elle est restée après l'indépendance considérée comme la banque des intérêts franco-mauriciens, par opposition à la State Bank. Ce rapport illustre les mécanismes de contre-pouvoir existant à Maurice entre secteurs public et privé. Il est significatif à cet égard de constater que, si l'actionnariat des deux banques est en réalité désormais fortement diversifié, le personnel de direction de la MCB est resté principalement francomauricien et, plus largement, issu de la Population Générale, tandis que celui de la State Bank est majoritairement indo-mauricien (comprenant des Hindous et des Musulmans).

19 - Le musée tire d'ailleurs son nom du timbre-poste éponyme, un rarissime bleu indigo de 2 pence, l'un des tout premiers en circulation dans l'île.

20 - Sur ce site, le sucre était déchargé du train à dos d’hommes pour être chargé par le même moyen sur les navires pour l'exportation. 
celles des entrepôts et de l'observatoire météorologique). Les bâtiments des docks et la gare du chemin de fer, qui auraient pu témoigner d'une mémoire créole (car les dockers appartenaient majoritairement à ce groupe du fait de l'ethnicisation des occupations professionnelles dans la société coloniale), et surtout du caractère douloureux de celle-ci, n'ont pas été conservés sur le site. Le musée lui-même, construit dans l'ancien quartier général des docks, ne signale pas ce pan d'histoire.

C'est donc avant tout une vision coloniale de l'histoire qui est valorisée dans le Blue Penny Museum et, par extension, sur le site tout entier du Caudan Waterfront : une histoire de la fondation de l'île à travers les grands axes historiques du développement des échanges avec l'extérieur, mais aussi à travers le recours au mythe de fondation tel qu'il est exprimé par la statue de Paul et Virginie ${ }^{21}$. Il s'agit ainsi de montrer «la relation entre qui nous sommes et d'où nous venons", dans le but de "rassembler les gens qui viennent de tous les horizons autour d'un point commun qui est le territoire d'accueil $»^{22}$.

La conception de l'histoire mauricienne présentée à travers le Blue Penny Museum s'inscrit ainsi dans le prolongement d'une mytho-histoire franco-mauricienne, témoignant de la conception d'une centralité du groupe dans la nation mauricienne et simultanément d'une conception de l'histoire nationale comme extensive de celle du groupe.

Cette même dialectique est présente dans l'écomusée de l'Aventure du sucre, construit en 2002 sur le site de l'usine sucrière de Beau Plan, dans le nord de l'île. L'objectif de l'écomusée, subventionné par les groupes sucriers Harel Frères, DRBC et Constance, n'était pas de faire une simple histoire des technologies sucrières à Maurice, mais de montrer comment le sucre a contribué à la genèse de la société, voire de la nation mauricienne.

L'exposition présente l'histoire mauricienne comme une conjonction de celle des différents groupes ethniques qui se sont implantés successivement dans l'île autour de l'activité sucrière. Le musée, en se voulant «[...] un miroir dans lequel tous les Mauriciens se retrouvent, qu'ils soient ancien administrateur sucrier descendant de colon français, ancien petit planteur descendant de laboureur, ou employé du secteur public dont les parents sont issus de la première immigration ${ }^{23}$ ", véhicule ainsi l'image d'une nation qui s'est littéralement construite sous la cheminée de l'usine sucrière. Contrairement au Blue Penny, l'Aventure du sucre consacre une place importante à l'esclavage et à l'engagisme, en montrant le rôle joué dans l'industrie sucrière par les esclaves et par les travailleurs engagés indiens qui les remplacèrent dans les champs de canne, pour conférer un sens social à cette souffrance ${ }^{24}$.

21 - L'histoire de Paul et Virginie a servi de mythe fondateur de la distinction raciale aux premiers temps de la colonie. S. Chazan-Gillig, «De l'abolition de l'esclavage à l'interprétation ethnique de la question nationale à l'île Maurice», Annuaire des pays de l'océan Indien, vol. 17, 2003, p. 337-340.

22 - Alain Huron, conservateur du Blue Penny Museum, entretien du 9 août 2007 avec Catherine Boudet au Blue Penny Museum, Port-Louis.

23 - Caroline Semonella-Martial, responsable communication du musée de l'Aventure du sucre, entretien du 27 août 2006 avec Julie Peghini à Beau Plan.

24 - L'histoire des docks, occultée au Blue Penny Museum, est d'ailleurs présente au sein de l'Aventure du sucre, à travers une exposition permanente. 
L'objectif du musée est donc clairement celui de la production d'une mémoire historique, au sens de Lavabre ${ }^{25}$, c'est-à-dire une tentative de proposer une interprétation du passé sucrier de manière à montrer et à légitimer la centralité de l'industrie sucrière dans la cohésion nationale. À partir du constat selon lequel les Mauriciens ne se sont pas encore approprié leur histoire et leur identité, cette production mémorielle est aussi production d'une mémoire collective, constituée des représentations partagées du passé. La visite du musée a pour vocation de produire in situ ces représentations partagées, cette référence à un vécu commun :

«Tout le monde à Maurice a un lien avec le sucre, directement ou indirectement [...] On parle du sucre et tout le monde s'y retrouve, et tout le monde est content de goûter la canne et le sirop de canne, car ce sont les mêmes souvenirs d'enfance par rapport au sucre. C'est un concept d'un sucre-plaisir qui ramène à un univers. Les Mauriciens se réapproprient le sucre et c'est une fierté pour eux ${ }^{26}$.»

L'usine sucrière, placée au centre de cette mémoire collective, affirme ainsi l'idéal d'un projet national «mauricianiste ${ }^{27}$ alternatif au modèle d' "unité dans la diversité», et dont le groupe franco-mauricien constituerait le cœur ethnique du fait de son rôle au sommet de la hiérarchie sucrière (par le quasi-monopole historique sur les usines centrales). Cette place centrale de catalyseur de l'unité nationale se retrouve en filigrane de la philosophie du musée, où il est affirmé que «ce sont les colons qui ont permis un destin extraordinaire ${ }^{28}$ ».

La mémoire de la "colonisation-fondation», dont la préservation s'accompagne d'une édulcoration du passé colonial, est prise à contre-pied par la politique mémorielle du gouvernement de l'État indépendant dominé par la bourgeoisie hindoue, pour qui le «moment-origine» est chronologiquement décalé dans le temps, et qu'il s'agit de s'approprier sur un mode dominant et non plus dominé.

\section{L’État et l'empilement des mémoires}

L'indépendance en 1968 marque, comme nous l'avons vu, la mise en place à Maurice d'un partage des pouvoirs aux termes duquel l'appareil d'État et le secteur public deviennent la prérogative de la bourgeoisie hindoue, tandis que le secteur privé, dont l'industrie sucrière est le moteur, reste dominé par les intérêts

25 - Nous retiendrons ici comme définition de la mémoire historique celle de Marie-Claire Lavabre, pour qui elle désigne les usages du passé et de l'histoire, tels que les groupes sociaux se l'approprient, par opposition à la mémoire collective, qui désigne des représentations partagées du passé. Cette appropriation peut en outre s'effectuer sur un mode dominé ou dominant. M.-C. Lavabre, «De la notion de mémoire à la production des mémoires collectives », in D. Cefaï (dir.), Cultures politiques, Paris, Presses universitaires de France, 2001, p. 242-244.

26 - Caroline Semonella-Martial, responsable communication du musée de l'Aventure du sucre, entretien du 20 juillet 2006 avec Catherine Boudet à Beau Plan.

27 - Le projet de "Mauricianisme» en tant que modèle de nation relève d'une tradition des élites politiques et intellectuelles franco-mauriciennes de la période coloniale britannique. Il consacrait la culture française comme haute culture, au sens de Gellner (Nations and Nationalisme, Paris, Payot, traduit de l'anglais par B. Pineau, 1983), c'est-à-dire comme assimilatrice des particularismes ethniques pour donner naissance à une nation unitaire.

28 - Caroline Semonella-Martial, entretien du 20 juillet 2006, déjà cité. 
franco-mauriciens. Dans ce partage des monopoles entre l'ancienne minorité dominante et la nouvelle majorité dominante, les autres communautés s'inscrivent dans une relation clientéliste : tandis que la communauté musulmane bénéficie des solidarités avec l'élite hindoue pour une place au sein du secteur public, le secteur privé constitue le principal bassin d'emploi de la population créole. Constitutionnellement, le mécanisme électoral du best loser ou «meilleur perdant» (voir note 1) consacre le principe de représentation des minorités ethniques au sein du législatif. Toutefois, ce principe d'équilibre de la représentation ethnique dans le politique n'empêche pas une vassalisation des communautés à l'égard de l'ethnicité hindoue majoritaire. La prééminence comme maître de la machine gouvernementale et parlementaire (en vertu du modèle westminstérien) du Premier ministre ${ }^{29}$, qu'une règle tacite à Maurice veut issu de la communauté hindoue ${ }^{30}$, est symptomatique de cette ethnicisation de l'appareil étatico-bureaucratique.

Le volet culturel et symbolique du modèle national mauricien s'exprime néanmoins dans le slogan de l' «unité dans la diversité», qui proclame la préservation des différences culturelles des groupes ethniques et fonde la légitimité de ces derniers au sein de l'ensemble national sur la valorisation d'un rattachement à une culture ancestrale. Cette posture s'inscrit en réaction contre la perspective assimilationniste du Mauricianisme prôné durant la période coloniale par les élites franco-mauriciennes et leurs alliés créoles, qui avaient milité contre l'indépendance avec l'argument qu'une nation unitaire était le pré-requis nécessaire à l'indépendance : «lorsque tous les Mauriciens sauront lire, lorsque toutes les communautés mauriciennes ne feront qu'une seule famille, alors, et alors seulement, on pourra avoir un gouvernement responsable à suffrage universel», avait déclaré Jules Koenig, le leader franco-mauricien du Parti mauricien social-démocrate en juin 1955 lors d'un meeting politique (Advance du 24 juin 1955). À l'inverse, pour Seewosagur Ramgoolam et les leaders hindous du Parti travailliste, l'indépendance devait marquer la naissance de la nation mauricienne. Le «moment-origine» de la nation réside dès lors dans l'octroi de l'indépendance, comme l'affirmait le journal travailliste Advance : "[...] On devrait octroyer à Maurice son indépendance le plus tôt possible. Dans son sillage suivra l'émergence d'une nation mauricienne - un besoin qui se fait ressentir de plus en plus ${ }^{31}$.»

L'inspiration politique du modèle indien permet de fixer l'indépendance comme moment-origine de la nation, appropriable sur un mode dominant, tout en capitalisant sur le moment-origine du groupe ethnique que constitue l'engagisme, mais dont l'appropriation restait alors dominée. Le choix de la date de l'indépendance est significatif de la production d'un registre mémoriel dominant par le rattachement à l'ancestralité indienne :

«Ce 12 mars est loin d'être un hasard du calendrier. Cette date qui marque l'acte de naissance de notre nation relève de tout un symbolisme de libération. Sir Seewosagur

29 - "Constitution. La quadragénaire aux 26 liftings», édition spéciale «40 ans d'indépendance» de L'Express de Maurice, mars 2008.

30 - Nazim Esoof, «Du droit de devenir Premier ministre», L'Express de Maurice, 24 mars 2008.

$31-«[\ldots]$ Mauritius should have its independence as soon as possible. In its train will follow a Mauritian nation - a need which is being felt more than ever." (Advance, 2 juillet 1964). 
Ramgoolam a délibérément choisi cette date pour rappeler la longue marche du sel du Mahatma Gandhi ${ }^{32}$.»

Le modèle d' «unité dans la diversité» aura dès lors vocation à préserver les spécificités culturelles qui avaient été menacées par l'hégémonie coloniale. En corollaire, la gestion du passé colonial se fait sur le mode d'une absence de politique de conservation, d'autant qu'alors la priorité est donnée au développement économique. Ce qui conduit dans les faits à un effacement de la mémoire coloniale, comme l'illustrent les aléas subis par le musée de la Photographie. Ce musée privé avait été créé en 1996 par un collectionneur issu de la Population Générale, Tristan Bréville, avec l'appui du maire de Port-Louis de l'époque, Jérôme Boulle. Installé au cœur de la capitale dans une demeure coloniale datant du XVIII ${ }^{\mathrm{e}}$ siècle, ce musée exprime une philosophie de conservation relevant du registre mémoriel de la "colonisationfondation", la mémoire nationale y étant donnée à voir sous l'angle d'une "haute culture», essentiellement du point de vue du développement des techniques, que ce soit la photographie, le chemin de fer ou l'aviation. Après le départ de Jérôme Boulle, le dernier maire issu de la Population Générale de la municipalité de Port-Louis, ses successeurs refusent treize années durant d'octroyer des subventions à Tristan Bréville pour son musée, tentant même à l'occasion de le déloger. En janvier 2008, lors de la construction d'un immeuble de dix-huit étages derrière le musée, la chute d'un panneau de vitres perfore son toit et détruit un millier de négatifs sur verre ainsi que toute la collection de films documentaires sur Maurice qu'il abritait. Cette atteinte au patrimoine photographique qu'abritait le musée a été rendue possible par l'absence de législation en matière de protection et de conservation patrimoniale.

L'attitude passive de l'État vis-à-vis du patrimoine, se traduisant par une politique patrimoniale lacunaire et par une absence de technicité en matière de conservation, se limite à la conservation de reliques de l'histoire naturelle et de monuments à la mémoire de personnages célèbres :

«Ils nous rappellent ces personnalités qui, à un moment ou un autre ont influencé ou forgé la destinée de notre pays, et nous incitent à leur rendre hommage. En même temps, nous ne devons pas oublier les coutumes et traditions que nos ancêtres nous ont transmises et que nous devons préserver jalousement comme des trésors culturels et nationaux ${ }^{33}$. »

Ces monuments personnifient les apports des communautés ethniques à la construction nationale $e^{34}$. À travers eux, la nation est ainsi conçue comme le

32 - Propos de Navin Ramgoolam, actuel Premier ministre et fils de Sir Seewosagur Ramgoolam, le premier Premier ministre de l'île indépendante. Navin Ramgoolam, "Unité j'écris ton nom", L'Express de Maurice, édition spéciale " 40 ans d'indépendance», mars 2008.

33 - "They remind us of and cause us to pay tribute to those personalities who, at a time or another, have influenced or carved the destiny of our country. At the same time we must also not forget the customs and traditions which our forefathers have bequeathed to us and which we must jealously guard as our national and cultural treasures. " (A. Parsuramen, ministre de la Culture, "Avant-propos», National Monuments of Mauritius, Rose Hill, Éditions de l'océan Indien, 1988.)

34 - Parmi les plus significatifs: Mahé de Labourdonnais, considéré comme le fondateur de la colonie; Adrien d'Épinay, responsable de l'obtention de la liberté de la presse en 1832; Rémy Ollier, journaliste "de couleur» ayant milité pour l'obtention des droits politiques pour les hommes de 
produit de la contribution des différents groupes ethniques. Un exemple en est donné avec le Silambou, monument érigé au cour de la ville commerçante de Rose Hill sous la pression de leaders des associations socio-culturelles tamoules : symbolisant l'île Maurice posée sur une fleur de lotus, ce monument est dédié à la mémoire des premiers Tamouls arrivés dans l'île comme artisans. En corollaire, la gestion étatique des spécificités culturelles passe par un marquage de l'espace par les références symboliques et religieuses des groupes respectifs. Les Mauriciens, en l'absence de référent collectif à vocation pan-ethnique, se retrouvent dès lors dans l'impossibilité d'investir le fond patrimonial commun d'une valeur symbolique, de se l'approprier de façon collective ${ }^{35}$.

Entre 1997 et 2003, un nouveau cadre juridique traduit la volonté de donner au patrimoine, désormais qualifié $\mathrm{d}^{\prime}$ "héritage national», un rôle de ciment d'une appartenance nationale ${ }^{36}$. La politique muséale est amendée dans ce sens, un Conseil des musées de Maurice étant créé en 2000 par voie législative (Mauritius Museum Council). Concrètement, dans les musées d'État, la volonté affichée de soutenir l'élaboration d'une mémoire collective se traduit par des pratiques d'«empilement». Ainsi, le musée de Mahébourg, fondé en 1950, est rebaptisé en 2000 "Musée national d'histoire de Mahébourg».

Ce changement de nom préfigure un changement d'orientation dans la vision du musée. Ce dernier était à l'origine un musée naval, retraçant l'histoire de la colonisation de l'île par les Hollandais, les Français et les Anglais, et réunissant une collection sur la bataille historique de Grand-Port ${ }^{37}$. Il est installé dans le château Gheude, ancienne demeure de la famille de Robillard (rachetée par l'État colonial en 1947), où ont été soignés en même temps les deux généraux protagonistes de la bataille, l'anglais Willoughby et le français Duperré ${ }^{38}$. Lors de la rénovation en 2000, il prend le nom de Musée national d'histoire de Mahébourg. À cette

couleur en 1846; Anjalay, jeune femme hindoue tombée sous les coups de feu de la police lors des révoltes des travailleurs sucriers en 1937.

35 - Il semblerait que, tout en étant capables d'identifier l'existence d'un fonds patrimonial commun dans des monuments commémoratifs, des bâtiments historiques ou des monuments publics tels que l'Hôtel du Gouvernement ou le Marché central, les Mauriciens ne les considèrent pas comme représentatifs de leur identité, laquelle s'inscrit davantage dans les édifices religieux, les fêtes et autres manifestations cultuelles, destinées à perpétuer les cultures ancestrales. J. M. Jauze, «Tourisme et construction identitaire : exemple de l'île Maurice dans l'océan Indien», in P. Frustrier (dir), Les identités insulaires face au tourisme, La Roche-sur-Yon, Siloë, 2007, p. 159-160.

36 - En 1985, un premier National Monuments Act dont le champ d'action était la "protection des structures d'intérêt historique, scientifique, artistique et esthétique» avait remplacé la politique patrimoniale datant de la colonisation qui relevait du Ancient Monuments Act de 1944, dont l'action se réduisait à la simple conservation des monuments. En 1997, un National Heritage Trust Fund Act $n^{\circ} 27$ assigne la gestion de l'«héritage national» au National Heritage Fund (NRF). Cet Acte est amendé en 2003 afin d'inclure des actions de sensibilisation du public au patrimoine national.

37 - La bataille navale du Grand-Port s'est terminée par la victoire de la flotte française sur la marine britannique en août 1810 (la seule victoire maritime des guerres napoléoniennes), quatre mois avant la capitulation de l'île.

38 - Ce musée témoigne donc lui aussi de l'étroite imbrication de l'histoire coloniale et de celle du groupe franco-mauricien, pour qui cette scène a préfiguré la réconciliation identitaire entre les influences anglaise et française de l'île. 
occasion, des œuvres et documents sur l'esclavage et sur l'engagisme, ainsi que des documents sur l'île Rodrigues y sont rajoutés ${ }^{39}$, dans l'optique de proposer une nouvelle interprétation de l'histoire coloniale.

Les anciennes collections sont aujourd'hui dans un état de dégradation avancée, y compris celles qui avaient été rénovées en 2000, à l'exemple du portrait de Mahé de Labourdonnais qui reste affligé d'un trou ${ }^{40}$, symbole s'il en est du double registre sur lequel s'exerce le rapport à la mémoire coloniale: un processus d'effacement passif doublé d'un «empilement» des mémoires. Ce double processus d'effacement/ empilement a vocation de rupture avec l'histoire coloniale considérée comme prénationale, et dont la mémoire de l'«indépendance-origine» cherche à s'extraire : «Moins on préserve, plus on disperse, et plus grand devient [l']espoir de l'avènement d'une île Maurice exempte de tout vestige colonialiste ou pire oligarchique», commente amèrement Yvan Martial, chroniqueur culturel dans L'Express de Maurice ${ }^{41}$.

La même logique d'empilement des mémoires se retrouve au Jardin botanique de Pamplemousses. Créé par Pierre Poivre en 1770, ce jardin d'acclimatation avait pour vocation de centraliser l'introduction dans l'île de variétés de plantes tropicales et leur diffusion vers les îles voisines. Pendant la période anglaise, il devient un lieu d'importation, de sélection et de distribution de nouvelles variétés de canne à sucre. À l'indépendance, le jardin est rebaptisé «Jardin botanique Sir Seewoosagur Ramgoolam», du nom du premier Premier ministre de l'île indépendante. Un samadhi (mémorial) consacré à la mémoire de ce dernier y est érigé, devant le château de Mon Plaisir, résidence construite en 1820 par les Anglais. L'attribution d'un nouveau nom et d'une nouvelle institution ont vocation à « changer le rituel » du lieu, ce contre quoi le paysagiste français Gilles Clément, sollicité pour son expertise, tire la sonnette d'alarme ${ }^{42}$. Lorsqu'il est question de construire un deuxième samadhi pour la commémoration en 2000 du centième anniversaire de la naissance de Sir Seewoosagur Ramgoolam, Gilles Clément dénonce l'incohérence esthétique d'un tel projet :

«Le projet (actuel) d'un samadhi encore plus monumental occupant l'espace central au devant du château paraît totalement aberrant et déplacé [...] Vouloir juxtaposer cet édifice en un lieu de mémoire antérieure à celui de la naissance de la nation - et connu comme tel par le monde - n'est ni judicieux, ni politiquement habile. Esthétiquement, il s'agirait d'un désastre.» (Gilles Clément, interview dans Week-End du $1^{\mathrm{er}}$ août 1999)

La construction du samadhi dans le Jardin s'inscrit dans la démarche classique d'empilement des mémoires, à des fins d'imposition, par-dessus la mémoire coloniale, d'une mémoire nationale. Mais par la connotation ethnico-religieuse qu'introduit le samadhi, cette mémoire nationale en vient à se confondre avec la mémoire ethnique de la communauté dont est issu le Premier ministre.

39 - Aline Groëme-Harmon, «Sous le vernis, un musée... », L'Express de Maurice, 18 mai 2007.

40 - Interview d'Emmanuel Richon, restaurateur d'œuvres d'art et ancien conseiller culturel du ministère de la Culture, dans L'Express de Maurice du 17 septembre 2007.

41 - Yvan Martial, «Qui sauvera notre patrimoine?» L'Express de Maurice du 19 juin 2006.

42 - Interview de Gilles Clément dans Le Mauricien du 13 février 1999. 
Alors que les élites hindoues ont pu, en s'appuyant sur l'appareil d'État, s'approprier sur un mode dominant une histoire vécue initialement sur un mode dominé, tel n'a pas été le cas des Créoles. La mémoire de l'esclavage n'est que très récemment apparue comme une catégorie de discours dans l'espace politique mauricien, ce qui témoigne de la position défavorisée que continue d'avoir le groupe créole dans le système sociopolitique.

\section{Les Créoles et la réappropriation de la mémoire de l'esclavage}

La mémoire de l'esclavage, d'une part édulcorée par les tenants du modèle francomauricien et d'autre part négligée par l'État ${ }^{43}$, est restée un tabou pour la communauté créole qui n'a pas pu s'en prévaloir pour se construire une identité positive. Ainsi, contrairement à la mémoire reposant sur le "colonisation-fondation » et à celle reposant sur «l'indépendance-origine», l'esclavage n'a pas pu donner lieu à une mémoire fondatrice.

Les dépossessions identitaires, culturelles et surtout mémorielles induites par la traite et l'esclavage ont eu pour effet «d'interdire le maintien du sujet (ici, collectif) comme producteur de son histoire ${ }^{44} »$. Dans un système politique mauricien qui pose la communauté ethnique et sa mémoire comme fondement principal de la légitimité de l'accès aux ressources de l'État, le groupe créole, considéré comme celui qui n'avait rien su préserver de sa culture ancestrale, et donc rien apporté à la nation, a été marginalisé, rendu incapable par le système même de promouvoir son identité ${ }^{45}$, qui a ainsi été définie en creux et stigmatisée. Pour décrire cette situation d'exclusion sociale, politique et économique des Créoles, un terme est communément employé depuis sa diffusion en 1993 par le prêtre catholique Roger Cerveaux : celui de malaise créole. L'expression marque ainsi «un des temps forts de la prise de conscience à échelle nationale d'un fait identitaire que les stigmates de l'histoire avaient contribué à refouler pendant longtemps ${ }^{46}$ ".

Dans la foulée, l'ethnonyme "Afro-Mauricien» est forgé par l'historien Gaëtan Benoît ${ }^{47}$ et d'autres intellectuels issus de ce groupe. Cette démarche de "réinvention

43 - Selon Emmanuel Richon, «Maurice néglige volontairement des pans entiers de la culture locale des populations (langue créole, musique séga, esclavage, patrimoine ou architecture vernaculaire)» («Les musées, alternative démocratique au communalisme culturel», http://potomitan. info/bio/richon.php).

44 - C. Chivallon, «L'usage politique de la mémoire de l'esclavage dans les anciens ports négriers de Bordeaux et Bristol», in S. Dufoix et P. Weil, L'esclavage, la colonisation et après, Paris, Presses universitaires de France, 2005, p. 562.

45 - Ce groupe, issu de la population esclave, n'a pas eu, après l'abolition de l'esclavage, accès aux mécanismes de la mobilité sociale, celle-ci s'étant effectuée à travers le métissage garant du «blanchiment», donc sur une base individuelle. Les membres du groupe connaissant une certaine réussite économique «montaient» dans le groupe "de couleur» et rejetaient alors toute identification aux Créoles, dont le phénotype négroïde incarnait le lien le plus fort avec le passé esclave. Ainsi, le terme de «créole» à Maurice implique un amalgame entre la couleur de peau (absence ou quasi-absence de métissage avec l'Européen) et la classe sociale (prolétariat). T. Arno et C. Orian, Île Maurice : une société multiraciale, Paris, L'Harmattan, 1986, p. 37.

46 - A. Carpooran, Île Maurice des langues et des lois, Paris, L'Harmattan, 2003, p. 10.

47 - G. Benoît, The Afro-Mauritians : An Essay, Moka (Maurice), Mahatma Gandhi Institute, 1985. 
d'une africanité ${ }^{48}$ » vise, en requalifiant le groupe dans une logique de symétrie avec les autres groupes de la société mauricienne (Indo-Mauriciens, Sino-Mauriciens, Franco-Mauriciens), à le situer dans la nation avec une identité ethniquement définie par son ancestralité.

Les émeutes de 1999, déclenchées par la mort en prison du chanteur créole Kaya, victime de violences policières, ont constitué le principal détonateur de ce processus de revendication mémorielle et identitaire. Elles ont surtout contribué à sa visibilité dans l'espace public mauricien. Ces émeutes, qui mettaient à mal l'image iréniste de la «nation arc-en-ciel» et dévoilaient la colère des Créoles contre le système ${ }^{49}$, ont obligé l'État mauricien à prendre en considération le malaise créole. Elles marquent ainsi une étape décisive : celle de sa politisation, qui donne lieu à une "accélération de la mémoire». Ce qui souligne le triple enjeu dans lequel est prise la construction de l'esclavage en tant que registre mémoriel : en premier lieu, la nécessité politique de rompre avec la posture du silence; en deuxième lieu, l'efficacité du registre de la mémoire de l'esclavage pour le positionnement communautaire; enfin, l'action mémorielle qui permet l'accomplissement des modalités de constitution identitaire $^{50}$ et à terme, d'organisation pour la lutte contre l'exclusion.

La rupture avec le tabou et le silence est venue non pas de l'État, mais de l'Église catholique, lorsque cette dernière reconnut officiellement l'iniquité du système esclavagiste et le rôle qu'elle a joué dans son maintien (en particulier par l'évangélisation forcée des esclaves). Le $1^{\mathrm{er}}$ février 2001 la hiérarchie de l'Église catholique demande officiellement pardon pour l'esclavage. Cette reconnaissance donne l'impulsion à la constitution de la mémoire de l'esclavage comme catégorie de discours, qui à son tour favorise l'accomplissement de nouvelles modalités de la définition identitaire et mémorielle des Créoles.

Le rastafarisme, introduit à Maurice par Kaya, cherche à doter l'identité créole d'une connotation plus positive par l'appropriation d'une mémoire ancestrale africaine sur un mode dominant (pour les Rastas, le pays mythique qui représente l'origine est l'Éthiopie d'Haïlé Sélassié) et non plus dominé par la référence à l'Afrique de l'esclavage. Cette position militante s'exprime principalement à travers le seggae, mouvement musical inventé par Kaya, un mélange de séga (la musique traditionnelle mauricienne héritée de l'esclavage) et de reggae. Les héritiers musicaux de Kaya, perpétuant cet idéal trans-ethnique du seggae en faveur d'une nation

48 - J.-L. Alber, «Les ressorts d'une africanité réinventée à Maurice», in D. Dimitrijevic, Fabrications des traditions, inventions de la modernité, Paris, Éditions de la Maison des sciences de l'homme, 2004, p. 107.

49 - Alors que les violences s'étendaient à l'île tout entière, les symboles du pouvoir d'État furent pris pour cibles par les émeutiers : postes de police, bureaux de poste, le siège de la télévision nationale, la maison d'un membre du gouvernement. En outre, par le saccage des centres économiques (usines de la zone franche, hôtels, grandes surfaces, agences bancaires), les émeutes exprimaient également la revendication d'un accès plus favorable aux ressources nationales. C. Boudet, "Émeutes et élections à Maurice. La mort de Kaya, aléa ou échec de la construction nationale?» Politique Africaine, $\mathrm{n}^{\circ} 7$ : Pouvoirs sorciers, 2000, p. 155.

50 - C. Chivallon, «L'usage politique de la mémoire de l'esclavage...», chap. cit., p. 576. 
trans-ethnique et d'une créolité dégagée des barrières de l'ethnicité ${ }^{51}$, soulignent le décalage qui peut exister entre les usages politiques de la mémoire, pris dans le moule ethnicisé du modèle de l' "unité dans la diversité», et les logiques d'appropriation de la mémoire collective du groupe qui au contraire suivent un idéal de plus en plus transculturel, désethnicisé ${ }^{52}$.

À la mort du chanteur, le mouvement rasta connaît un regain d'engouement parmi la jeunesse créole. Son principal fer de lance s'appelle l'Association socio-culturelle rastafari (ASCR). Créée en 1999, elle a pour double objectif d'améliorer l'image de soi des Afro-Créoles et de les faire sortir des cités-ghettos qu'ils occupent. En support à cet objectif, l'ASCR et son président, José Rose, participent à une démarche de réhabilitation d'une histoire "par le bas" de l'esclavage, qui prenne en compte les souffrances et les maltraitances subies par les esclaves. Cette réhabilitation s'appuie sur la collecte de l'histoire orale et des légendes de l'esclavage, liées en particulier à la montagne du Morne ${ }^{53}$ :

"Je suis impliqué avec un historien, Norbert Benoît, pour récolter l'histoire créole. Elle n'a jamais été écrite, seule le côté blanc l'a été... [...] Toute la mémoire des descendants d'esclaves n'a pas été conservée par écrit : les sites où on a exécuté les esclaves, la place de l'exécution à Plaine-Verte, on n'en parle pas, cela ne se sait que de mémoire orale. De même Congoma, Pamplemousses, Belle-Mare (là-bas, il y a une prison où les esclaves devaient se tenir debout, sans bouger), Rivière Noire, Souillac, le Morne ${ }^{54}$.»

À travers la réaffirmation de l'héritage oral, c'est l'écriture d'une contre-histoire que revendique l'ASRC :

«L'histoire est mal racontée dans les manuels scolaires. Sur le Morne, il est dit que les Marrons se sont jetés [de la montagne dans le vide (NDA)] quand les Anglais sont venus les prévenir de l'abolition de l'esclavage. Le Cernéen a relaté cette histoire le premier au début du siècle. Cela justifie que les esclaves sont des idiots, qui interprètent mal l'histoire des autorités. Mais c'est faux, ce n'est pas possible de croire en cette histoire-là sur le Morne. La véritable histoire est donnée par Nagapen, qui explique que les Marrons étaient déjà presque tous morts et que les autorités le sachant sont allées faire une battue. Il est intéressant de comprendre que c'est la première histoire qui a été promue, dans laquelle les esclaves sautent comme des idiots quand ils sont libres ${ }^{55}$ !»

La production de cette contre-histoire est, après l'invention d'un ethnonyme, la deuxième expression du processus de "renversement du stigmate ${ }^{56}$ " attaché à la mémoire de l'esclavage. La figure de l'esclave marron cristallise ce renversement : alors qu'elle était présentée dans la vision coloniale comme une menace à la stabilité du système, elle est désormais revalorisée pour connoter la fierté et

51 - Voir C. Boudet, «Émeutes et élections à Maurice...», art. cit., 2000, et S. Chazan-Gillig, «De l'abolition de l'esclavage à l'interprétation ethnique de la question nationale à l'île Maurice», art. cit., 2003.

52 - Kaya se revendiquait métis et concevait le seggae non pas comme la musique du groupe créole, mais comme la musique de tous les Mauriciens.

53 - Selon les récits de la tradition orale, jusqu'à présent non encore confirmés par des fouilles archéologiques, la montagne aurait abrité des esclaves en fuite, dits «Marrons». Sommet inaccessible, elle les aurait ainsi protégés des chasseurs de Marrons.

54 - José Rose, président de l'ASCR, entretien du 3 août 2007 avec Julie Peghini à Sable Noir.

55 - Idem.

56 - M. Wieviorka, Le racisme, une introduction, Paris, La Découverte, 1998. 
la liberté. Par la relecture du passé, la figure fondatrice du Marron, incarnant la résistance contre le système esclavagiste, acquiert valeur de mythe par son caractère d'exemplarité. Malgré la «dépossession comme point de départ ${ }^{57}$ » qui avait rendu impossible l'émergence de mythes fondateurs, le marronnage fournit désormais la possibilité pour les Créoles de forger à leur tour une mytho-histoire orientée à la fois vers la connaissance de la vérité et vers la revendication d'une justice sociale. Elle répond à la recherche d'une légitimité historique, préalable indispensable à l'accession à une légitimité politique.

L'Organisation fraternelle/les Verts des frères Sylvio et Élie Michel témoigne du passage au politique de cette revendication mémorielle autour de l'esclavage. L'organisation, bien que non représentée dans l'espace partisan mauricien, est revendiquée comme un parti politique par ses leaders. Elle a notamment obtenu de l'État mauricien en 2001 que l'esclavage soit commémoré à Maurice comme une fête nationale, avec comme date symbole le $1^{\mathrm{er}}$ février, qui est désormais jour férié. Elle continue depuis à militer pour l'octroi d'une compensation aux descendants d'esclaves.

Cette revendication a obtenu de la scène internationale - notamment avec la reconnaissance de la traite négrière comme crime contre l'humanité, et des projets comme celui de La route de l'esclave mis en place par l'UNESCO en 2000 - la légitimité qu'elle n'avait pas auprès de l'État mauricien. Alors que les frères Michel revendiquaient depuis 1975 une commémoration officielle de l'abolition de l'esclavage, il faut attendre 2001 pour que celle-ci soit agréée par l'État. De même, c'est en février 2005, à la suite d'une déclaration de la Commission de l'Union africaine, que la question d'une compensation aux descendants d'esclaves, pourtant revendiquée par l'Organisation fraternelle/les Verts, est posée devant le Parlement mauricien.

La politisation du discours sur l'esclavage, venant de l'extérieur de la scène mauricienne, rend la création de mécanismes pour la représentation de la population créole mauricienne impératifs ${ }^{58}$. La réingénierie de l'identité créole via la mémoire de l'esclavage débouche sur des revendications égalitaristes. Deux associations de défense des Créoles, la Fédération des Créoles mauriciens (FCM), créée en septembre 2007 par un prêtre catholique, Jocelyn Grégoire, et L'Union chrétienne (LUC), présidée par l'historien Benjamin Moutou, protestent que la fonction publique ne compte que $1,5 \%$ de Créoles, alors que ce groupe constitue $35 \%$ de la population. Ils revendiquent en conséquence une représentation proportionnelle à son nombre ${ }^{59}$, allant jusqu'à préconiser la mise en place d'une politique d'affirmative action (de "discrimination positive») à cet effet ${ }^{60}$. La FCM et L'Union chrétienne réclament également une modification de la Constitution

57 - C. Chivallon, «L'usage politique de la mémoire de l'esclavage...», chap. cit., p. 562.

58 - A. Prabhu, «Representation in Mauritian Politics : Who Speaks for the African Pasts ?» International Journal of Francophone Studies, vol. 8(2), 2005, p. 195.

59 - "Père Grégoire: "Il faut 35 \% de Créoles fonctionnaires" ", L'Express de Maurice du 26 septembre 2007.

60 - «Quelle égalité des chances pour les Créoles ?» L'Express de Maurice du 24 avril 2007. 
dans le sens d'une suppression de la catégorie "Population Générale» au profit d'une communauté des "Créoles et autres Chrétiens».

De même que la politisation de la mémoire de l'esclavage a permis de faire accéder le groupe créole à une identité positive d'acteur politique de l'espace politique mauricien ${ }^{61}$, inversement, cette restauration de la participation politique du groupe était une condition nécessaire pour en faire un agent actif de l'histoire ${ }^{62}$. Un certain équilibre est ainsi restauré par rapport aux deux autres modèles du triptyque mémoriel mauricien. Est-ce pour autant que ces mouvements de fond augurent de l'émergence d'une mémoire nationale partagée à Maurice?

\section{La réingénierie du triptyque mémoriel}

Les rapports entre registres mémoriels dessinent des zones de conflictualité ou de négociation qui sont tributaires à la fois du décalage temporel et politique entre moments-origine, des différents modes d'appropriation (dominant ou dominé) du passé et des rapports hégémoniques dans lesquels s'inscrivent les groupes ethniques au sein du modèle national. Si chacun des registres mémoriels a entrepris la construction d'une mémoire historique et d'une mémoire collective, se pose encore la question de l'émergence au plan national d'une mémoire collective au sens non plus seulement des usages politiques du passé, mais d'un travail d'homogénéisation des représentations de ce passé, celui-ci étant lié à la capacité d'intégration de la collectivité nationale ${ }^{63}$.

Le recours à l'antériorité comme mode de légitimation souligne la prégnance du modèle de la «colonisation-fondation" et sa capacité à influencer tout le champ mémoriel mauricien, qui reste étroitement orienté par cette conception de la légitimité en termes d'antériorité sur le sol de l'île et de contribution à l'histoire. Ainsi, le mythe du Marron emprunte au registre du «moment-fondation » quand l'historien Amédée Nagapen affirme que, même si aucune lignée des Marrons de l'époque hollandaise (1638-1710) n'a survécu jusqu'à la colonisation française (1715), ces premiers Marrons peuvent être considérés comme les premiers Mauriciens, autrement dit comme les véritables primo-arrivants ${ }^{64}$. Cette dépendance envers le registre dominant de la "colonisation-fondation" ${ }^{65}$ traverse même le champ de la recherche historiographique mauricienne, comme l'illustre la démarche de

61 - A. Prabhu, «Representation in Mauritian politics...», art. cit., 2005, p. 195.

62 - C. Poirier, "Mémoire collective, identité et politique. La société québécoise et sa relation problématique avec le passé», in I. Molinaro et C. Rolfe (éd.), Focus on Quebec 2. Further Essays on Quebecois Society and Culture, Le Greff, Edinburgh and Leceister, 2000, p. 83.

63 - M-C. Lavabre, "De la notion de mémoire à la production des mémoires collectives», chap. cit., 2001, p. 246.

64 - A. Nagapen, Le Marronnage à l'Isle de France - Île Maurice. Rêve ou riposte de l'esclave? PortLouis, Centre Nelson Mandela pour la culture africaine, 1999, p. 391.

65 - Cette perméabilité du champ mémoriel au modèle de l'antériorité ne s'exerce pas seulement pour la mémoire de l'esclavage. Le Silambou, monument dédié à la mémoire de la contribution des Tamouls à la fondation de la nation mauricienne, porte cette même trace en voulant souligner leur antériorité sur le sol de l'île par rapport au reste du groupe hindou majoritaire ayant pour langue ancestrale le hindi. 
l'historien Jocelyn Chan Low lorsqu'il s'oppose au mythe de «l'Européen bâtisseur» pour procéder à son renversement en affirmant que les "vrais bâtisseurs » ont été "des artisans de l'Inde et des esclaves " ${ }^{66}$.

Le registre mémoriel dominant de la "colonisation-fondation » s'articule au modèle politique de l' « unité dans la diversité» pour forger un rapport entre registres mémoriels qui se calque sur le rapport entre groupes ethniques fonctionnant suivant le modèle du «bien limité». Ainsi le père Jocelyn Grégoire justifie sa demande d'une meilleure représentativité des Créoles dans la fonction publique en s'appuyant sur l'argument selon lequel «[...] Un Créole est un Créole. Il a une contribution à apporter au pays et sa valeur culturelle doit s'émanciper pour le bien de tous. En tant que Créole, il a sa part dans la cuisson du gâteau national $[. . .]^{67}$ » et en conséquence il faut que "[...] l'existence d'une partie de la population qui a contribué à bâtir le pays, comme les autres, soit reconnue ${ }^{68} »$. Cette application du modèle interethnique du "bien limité» au champ mémoriel a pour effet d'entraîner des rapports de concurrence entre registres mémoriels.

Cette concurrence est particulièrement explicite dans la façon dont l'État traite les demandes de classement au patrimoine mondial de l'humanité par l'UNESCO de deux lieux très symboliques de l'histoire mauricienne: la montagne du Morne dans le sud de l'île, haut lieu du marronnage au temps de l'esclavage, et l'Aapravasi Ghat à Port-Louis, lieu de débarquement des premiers Indiens engagés sur l'île en 1834. Bien que le dossier du Morne ait été instruit avant celui de l'Aapravasi Ghat, c'est ce dernier qui obtint d'être classé "patrimoine mondial» le premier, l'État ayant mis en œuvre des moyens énormes pour ce faire. Ce processus de réhabilitation des mémoires des dominés relève donc moins d'une mise sur pied d'égalité, que d'une relation de symétrie concurrentielle, dans une logique de "lieu de mémoire créole versus lieu de mémoire indien ${ }^{69}$ ». Il est significatif à cet égard, que le Jardin de Pamplemousses, qui avait été désigné comme le premier site historique à classer au patrimoine mondial de l'humanité, ne l'ait finalement pas été, parce qu'il est considéré par l'État comme une construction coloniale ${ }^{70}$,

66 - «Il convient de rendre l'historiographie coloniale [...] support de l'ordre établi responsable de la vision arrangée de la réalité qui établit le mythe de l'Européen bâtisseur que cultiveront les descendants de colons à travers les musées, les monuments publics, la commémoration du $200^{e}$ anniversaire de Mahé Labourdonnais en 1899 [...]. Le fait que les vrais bâtisseurs soient des artisans de l'Inde et des esclaves sera occulté pendant plus d'un siècle», Jocelyn Chan Low, dans "Questions à Jocelyn Chan Low, historien : $1^{\text {er }}$ février, passage de l'amnésie collective à la mémoire affranchie», L'Express de Maurice du 29 janvier 2001.

67 - Interview de Jocelyn Grégoire dans Le Mauricien du 18 octobre 2007

68 - Interview de Jocelyn Grégoire dans Week-End du 4 novembre 2007.

69 - S. Carmignani, «Figures identitaires créoles et patrimoine à l'île Maurice, une montagne en jeu», Journal des anthropologues, vol. 104-105, 2006, p. 270.

70 - Pourtant, selon Jocelyn Chan Low, le Jardin de Pamplemousses aurait pu constituer à double titre un candidat idéal pour le classement par l'UNESCO : d'une part, la philosophie de protection de la nature qui avait présidé à sa fondation constituait à l'époque une première mondiale, d'autre part il est considéré par la population mauricienne comme un site appartenant à tous les Mauriciens, qui s'y rendent volontiers en pique-nique (entretien du 17 août 2007 avec Julie Peghini à l'université de Maurice). 
comme un site symbolisant principalement les Franco-Mauriciens, qui restent investis de l'image de "colonisateur interne ${ }^{71} »$.

La même logique d' "association-concurrence» des mémoires ${ }^{72}$ peut s'observer avec le timbre-poste réalisé pour la commémoration de 2001 (qui se voulait dans un premier temps conjointe), mettant en scène un engagé indien et un esclave qui avancent main dans la main vers un avenir radieux. L'engagé mène l'esclave par la main, comme s'il était venu le libérer, ce qui peut apparaître comme une manière subtile de s'accaparer l'histoire ${ }^{73}$. Les deux effigies semblent ainsi "endosser le projet collectif de construction de la nation mauricienne : sa marche ininterrompue vers la liberté, l'indépendance, la souveraineté, la République ${ }^{74}$ » mais, comme le souligne J.-L. Alber, les deux protagonistes figurent en fait une énonciation idéalisée, voire théâtralisée de la société mauricienne, masquant derrière une historicité romantique les rapports de pouvoir ethnique.

Alors que la mémoire hindoue capitalise sur une communauté de souffrance en s'associant à la mémoire de l'esclavage - le statut de victime procurant un argument de légitimité, un "privilège moral et politique ${ }^{75}$ » - cela contribue à dépouiller la communauté créole des spécificités ethniques susceptibles de fonder sa représentation égalitaire ${ }^{76}$. Ainsi, la confrontation des registres de mémoire se joue sur le mode de l'appropriation/dépossession qui est la caractéristique propre du modèle politique de l'«unité dans la diversité».

La réappropriation collective du passé colonial, engagée récemment par le gouvernement actuel, va de pair avec une politique de "démocratisation de l'économie». Ce projet politique se présente comme une "politique corrective d'un héritage négatif», par un élargissement du capital et de la propriété foncière et s'accompagne d'une politique mémorielle à visée égalitariste, "parce que nous voulons construire

71 - Le colonisateur britannique apparaissant à cet égard et en contrepoint, davantage comme "arbitre qu'agent des rapports coloniaux de production et de la rudesse des hiérarchies sociales" (Catherine Boudet, interview dans Le Mauricien du 7 juin 2007).

72 - Les associations socioculturelles effectuant un lobbying pour le compte des différents groupes ethniques ont joué un grand rôle dans cette concurrence entre mémoires. Ainsi, l'hebdomadaire Lavoix Kréol, porte-parole des intérêts de la communauté créole, dénonçait la priorité donnée à l'Aapravasi Ghat sur le Morne comme une atteinte à la dignité des Créoles : "Nous sommes en train de perpétuer l'insulte de faire croire que l'esclavage et l'engagisme c'est la même chose [...] Seriez-vous en train de perpétuer cette haine que vous avez reçue en héritage envers les descendants d'esclaves?» (Lavoix Kreol, n³52 du 21 au 24 juillet 2006.) De façon symétrique, lorsqu'en 2000, l'État a tenté d'instaurer une date conjointe pour la commémoration de l'abolition de l'esclavage et celle du début de l'engagisme, en choisissant le $1^{\mathrm{er}}$ février, il a dû se rétracter sous la pression des associations socioculturelles hindoues. Deux dates séparées de commémoration ont donc finalement été choisies : le $1^{\mathrm{er}}$ février pour l'abolition de l'esclavage, et le 2 novembre pour la commémoration de l'engagisme.

73 - Alain Huron, conservateur du musée du Blue Penny, entretien 16 août 2006 avec Julie Peghini au Blue Penny Museum, Port-Louis.

74 - J.-L. Alber, «Les ressorts d'une africanité réinventée à Maurice», chap. cit., 2004, p. 109.

75 - T. Todorov, Les abus de la mémoire, Paris, Arléa, 1995, p. 56.

76 - A. Prabhu, «Representation in Mauritian politics...», art. cit., 2005, p. 196. 
une nation où les descendants d'esclaves laisseront derrière eux l'humiliation et la rancour et feront de l'égalité un fait de la vie quotidienne ${ }^{77}$ ».

L'articulation entre la réappropriation mémorielle du passé colonial et la nouvelle politique de redistribution des ressources économiques (la seconde étant discursivement justifiée par la première) s'appuie sur l'esclavage comme moment-origine à vocation ontologique: en affirmant que «[l'esclavage] nous a laissé avec une distribution des richesses qui reste encore en faveur de ceux qui ont bénéficié de $l^{\prime}$ esclavage ${ }^{78}$ », tous les mécanismes ultérieurs de production des inégalités sociales sont ainsi oblitérés.

À nouveau, cette politique mémorielle de réappropriation d'une mémoire historique partagée s'effectue suivant le registre de l'appropriation-dépossession, cette fois-ci en direction du groupe franco-mauricien. Alors que la politique mémorielle à visée égalitariste s'appuie sur le renversement des valeurs anciennes attachées au passé colonial, ce sont les symboles propres à ce groupe qui sont renversés, mettant à mal son identification au projet national. Ainsi, le personnage historique d'Adrien d'Épinay, qui pour les Franco-Mauriciens constituait l'archétype même du Franco-Mauricien et le symbole de la construction de la nation mauricienne $e^{79}$, devient la cible privilégiée du renversement des valeurs. Sa statue, qui se trouve dans le Jardin de la Compagnie, en plein cœur de la capitale, a été d'abord menacée d'être déboulonnée, à l'occasion de la commémoration de l'abolition de l'esclavage, par des ministres du gouvernement. Le fer de lance de cette initiative était le ministre des Administrations régionales, James Burty David (issu de l'élite de couleur au sein du groupe de la Population Générale), qui apposa finalement une plaque sur la statue avec un poème sur la liberté, inspiré d'Éluard, à la date symbolique du $1^{\mathrm{er}}$ février 2007 :

"J'ai écrit un poème qui est sur le socle de la statue, pour que l'on sache au moins qui était d'Épinay, qu'il ne soit pas là à la même place que les autres, comme si de rien n'était... Ce poème sur la liberté, inspiré par Éluard, est destiné à la jeune génération, pour qu'elle sache. D'Épinay est le symbole de l'esclavagisme, alors que c'est l'esclave qui méritait de trouver une place dans ce jardin ${ }^{80}$.»

Dans un autre geste symbolique réalisé le jour précédent, le même ministre avait sectionné les clôtures de terrains à bail loués par l'État à des Franco-Mauriciens au pied de la montagne du Morne:

"Je l'ai fait en tant que ministre et en tant que défenseur de la liberté : c'est un acte fort pour revendiquer mon aspiration à la liberté pour le peuple mauricien. C'est un peuple qui ne connaît pas son histoire. Les racines sont importantes, pour voir la lutte qui est menée ${ }^{81}$.»

77 - «The Abolition of the Slavery and its Legacy in Mauritius», discours prononcé par le Premier ministre Navin Ramgoolam à Hull (GB), le 4 juin 2007.

78 - Idem.

79 - C. Boudet, Les Franco-Mauriciens entre Maurice et l'Afrique du Sud..., op. cit., 2004, p. 155.

80 - James Burty David, entretien du 22 août 2007 avec Julie Peghini au ministère des Administrations régionales, Port-Louis.

81 - L'Express de Maurice du $1^{\text {er }}$ février 2007. Quelques semaines auparavant, le ministère des Terres et du Logement avait entamé des procédures pour reprendre ces terres à bail à la société qui les louait, la société Morne-Brabant dirigée par le Franco-Mauricien Bertrand Giraud, qui avait pour projet d'y construire des villas dans le cadre d'un projet d'IRS (Integrated Resort Scheme). L'Express de Maurice du 5 janvier 2007. 
Le discours mémoriel subversif s'accompagne ainsi de discours identitaires virulents contre l'élite sucrière franco-mauricienne, comparée à une "classe de capitalistesdinosaures $^{82}$ » disposant de privilèges hérités d'un autre âge et qui ne seraient plus en accord avec la distribution des ressources qui «devrait» prévaloir. Ce discours d'ethnopolitique, qui par sa virulence, a été qualifié de white-bashing (ou «campagne anti-blancs») par la presse, vient appuyer la remise en question par le gouvernement des accords passés entre l'État et les sucriers franco-mauriciens au sujet de la conversion d'une partie des infrastructures de production du sucre de canne, en productions annexes notamment pour la production d'énergie (bagasse, éthanol). Ainsi, les usages discursifs de la mémoire ont vocation à soutenir la tentative de l'État de remettre en question le partage des pouvoirs hérité de la décolonisation. Le caractère instrumental de cet usage politique de la mémoire se révèle dans le décalage entre le discursif et la pratique, comme le souligne ironiquement un journal mauricien à propos de la rencontre, quelques mois après les épisodes de la statue d'Adrien d'Épinay et des clôtures du Morne, entre James Burty David et des décideurs économiques franco-mauriciens dans un grand hôtel mauricien :

\begin{abstract}
"Quant à James Burty David, on ne sait pas s'il a pénétré l'enceinte de cet établissement avec la statue d'Adrien d'Épinay dans l'espoir de trouver un lieu d'accueil alternatif au Jardin de la Compagnie d'où il veut la déloger ou s'il était muni de son sécateur pour couper quelques fils barbelés autour de l'hôtel cinq étoiles. Il aurait été intéressant, dans un régime qui pratique vraiment la transparence, de pouvoir filmer tout ce beau monde à la table des "gran missié-là". Mais non, nous avons affaire à de fieffés hypocrites qui ont un comportement et un discours lorsqu'ils sont devant tel auditoire et qui se cachent lorsqu'ils sont en train de festoyer avec le gros capital.» (Week-End du 23 septembre 2007)
\end{abstract}

Le potentiel subversif de la mémoire, sa capacité à remodeler la vision du passé pour une finalité relevant de l'intention politique - ici une politique à visée égalitariste entre groupes - contribue ainsi à masquer la réalité de la permanence des ententes consociatives. Le décalage entre le discursif et la pratique témoigne du décalage existant entre, d'une part, les logiques électoralistes et d'autre part, les ententes consociatives entre le secteur privé et l'État, malgré leur tentative de remise en question par ce dernier.

\title{
Conclusion
}

Le registre politique de «l'indépendance-fondation» se positionne par rapport à celui de la «colonisation-fondation» dans un rapport de rupture tandis qu'il entretient vis-à-vis de la mémoire de l'esclavage une posture fondée sur l'idée d'une communauté de destin et de souffrance. Depuis 2000, la réappropriation de la mémoire historique des "sans-voix» de l'histoire mauricienne est désormais mise au centre du travail de fondation d'une identité nationale, mais cela ne suffit pas à garantir l'émergence d'une mémoire nationale communément partagée.

La confrontation des registres de mémoire s'effectue sur le mode de l'appropriationdépossession qui est le propre du fonctionnement des relations interethniques dans

82 - Interview du ministre de la Justice, Rama Valayden, dans le Mauritius Times du 28 septembre 2007. 
le modèle de l'«unité dans la diversité», ce qui empêche fondamentalement la réalisation d'un équilibre mémoriel (non-hégémonique) entre les différents registres.

Tandis que le modèle national de l'«unité dans la diversité» se fonde sur la reconnaissance de toutes les communautés, l'appareil d'État reste fortement marqué ethniquement par une prépondérance de l'identité hindoue. Cette interférence a pour effet de rendre impossible la réalisation du mécanisme selon lequel «l'histoire [...] identifie la nation à l'État et leur procure une identité commune, une identité "national-étatique" reconnue comme légitime par la majorité des citoyens ${ }^{83}$ ». Ce ne serait donc pas tant par défaut d'histoire commune qu'une mémoire collective peine à émerger, mais inversement parce que l'usage politique fait par l'État du passé reste subordonné à un registre mémoriel hégémonique de type ethnique. Cette absence de référents collectifs (trans-ethniques) forts, cette impossibilité à suturer une identité "national-étatique», conduisent à une vision de la culture mauricienne comme étant toujours en devenir du fait de l'impossibilité de s'enraciner dans un passé commun : "We have une culture mauricienne spécifique, bien à nous et en devenir depuis longtemps déjà ${ }^{84}$.»

Conformément à son programme électoral énoncé en 2005, le gouvernement de Navin Ramgoolam met actuellement en place une commission Justice et Vérité, visant à produire "une évaluation des conséquences de l'esclavage et de l'engagisme durant la période coloniale et jusqu'à aujourd'hui ${ }^{85}$ ", ce afin "d'établir les faits dans le but de se réconcilier avec l'histoire» (propos du Premier Ministre cités dans L'Express de Maurice du 11 septembre 2007). Cette commission, inspirée du modèle de la commission Vérité et Réconciliation sud-africaine, mais appliquée au passé colonial, constitue une étape inédite dans le processus de gestion de la mémoire à Maurice. C'est de nouveau le "moment-origine» qui est convoqué, cette fois-ci pour expliquer les inégalités contemporaines : «nous avons besoin d'une commission de ce genre pour commencer une réflexion sur l'origine de tout un chacun et les handicaps que ces origines imposent", déclare le professeur Shell, l'historien sud-africain pressenti pour présider la commission, dans une interview accordée au Mauricien du 13 septembre 2007. En ce sens, encore une fois, la mémoire apparaît comme le versant symbolique de l'action politique, l'objectif de la commission à travers le travail de mémoire étant d'instaurer à terme, d'après Robert Shell, une "égalité parfaite et totale pour tous les Mauriciens» (L'Express de Maurice du 11 septembre 2007) et de contribuer par là consolider la nation mauricienne (L'Express de Maurice du 16 septembre 2007). En proposant à la fois la réalisation de recherches historiographiques sur des aspects encore peu ou pas étudiés de l'histoire de l'esclavage et de l'engagisme, et le témoignage des individus pour exposer leur sentiment quant à l'héritage colonial, le projet de commission aurait vocation à encourager la

83 - P. Ruiz Torres, «Les usages politiques de l'histoire en Espagne. Formes, limites et contradictions", in F. Hartog et J. Revel (dir.), Les usages politiques du passé, Paris, Éditions de l'École des hautes études en sciences sociales, 2001, p. 137.

84 - Déclaration du vice-Premier ministre, Public Bills, débat n 5 du 17 avril 2001.

85 - " $\ldots$ an assessment of the consequences of slavery and indentured labour during the colonial period up to the present», selon l'extrait du projet de loi cité dans L'Express de Maurice du $1^{\mathrm{er}}$ mai 2008. 
production d'une mémoire nationale à la fois historique et collective. À cet égard, la commission assumerait deux des trois fonctions essentielles que remplit le regard sur le passé: une fonction d'anamnèse critique et une fonction thérapeutique ${ }^{86}$, comme l'exprime Jocelyn Chan Low pour qui la commission "[...] permettra d'exorciser et de cicatriser les blessures du passé» (cité dans L'Express de Maurice du 16 septembre 2007). On peut néanmoins se demander comment la commission Justice et Vérité serait réellement en mesure de produire la troisième fonction du regard sur le passé, à savoir la possibilité d'«amnésie constructive» qui seule permet à une société de se forger une mémoire partagée ${ }^{87}$, si elle ne se dégage pas des usages politiques de la mémoire à des fins d'ingénierie ethnique.

86 - C. Poirier, «Mémoire collective, identité et politique...», chap. cit., 2000, p. 85.

87 - Ibid. 


\section{Bibliographie}

- Alber J.-L., «Les ressorts d'une africanité réinventée à Maurice», in D. DimitriJEviC, Fabrications des traditions, inventions de la modernité, Paris, Éditions de la Maison des sciences de l'homme, 2004.

- Arno T. et C. Orian, Île Maurice : une société multiraciale, Paris, L'Harmattan, 1986.

- Benoît G., The Afro-Mauritians : An Essay, Moka (Maurice), Mahatma Gandhi Institute, 1985.

- Boudet C., "Émeutes et élections à Maurice. La mort de Kaya, aléa ou échec de la construction nationale?» Politique africaine, $\mathrm{n}^{\circ} 79$ : Pouvoirs sorciers, 2000, p. 153-164.

- Boudet C., «L'émergence de la démocratie consociative à Maurice (1948-1968)", Annuaire des pays de l'océan Indien vol. 17, 2003, p. 325-336.

- Boudet C., Les Franco-Mauriciens entre Maurice et l'Afrique du Sud: identité, stratégies migratoires et processus de recommunautarisation, thèse de science politique, Sciences Po Bordeaux, 2004.

- Boudet C., «Pouvoirs et technologies en situation coloniale: les Franco-Mauriciens et le monopole des technologies sucrières à Maurice et au Natal (1825-1968)", Revue historique de l'océan Indien, $\mathrm{n}^{\circ} 2$, AHIOI, 2006, p. 178-193.

- Boudet C., «Une minorité ethnique dominante face à la décolonisation : l'émigration franco-mauricienne en Afrique du Sud (1947-68)", Journal of Mauritian Studies, New Series 3(1), 2006, p. 26-49.

- Boudet C., «Les Franco-mauriciens : une identité pollinisée», Revue européenne des migrations internationales, vol. 23 (3), 2007, p. 109-131.

- Candau J., Mémoire et identité, Paris, Presses universitaires de France, 1998.

- CARMignani S., «Figures identitaires créoles et patrimoine à l'île Maurice, une montagne en jeu", Journal des anthropologues, vol. 104105, 2006, p. 265-285.

- Carpooran A., Île Maurice des langues et des lois, Paris, L'Harmattan, 2003.
- Chan Low J., «Les enjeux actuels des débats sur la mémoire et la réparation pour l'esclavage à l'île Maurice», Cahiers d'études africaines, XLIV (1-2), $\mathrm{n}^{\circ} 173-174,2004$, p. 401-418.

- Chazan-Gillig S., «De l'abolition de l'esclavage à l'interprétation ethnique de la question nationale à l'île Maurice», Annuaire des pays de l'océan Indien, vol. 17, 2003, p. 337-347.

- Chivallon C., «L'usage politique de la mémoire de l'esclavage dans les anciens ports négriers de Bordeaux et Bristol», in S. Duforx et P. WeIL, L'esclavage, la colonisation et après, Paris, Presses universitaires de France, 2005, p. 559-584.

- Foster G., «Peasant Society and the Image of the Limited Good", American Anthropologist, $\mathrm{n}^{\circ} 4,1967$, p. 293-315.

- Gellner E., Nations et nationalisme, Paris, Payot, trad. de l'anglais par B. Pineau, 1983.

- Hoetink H., «Resource Competition, Monopoly and Racial Diversity", in L.A. Despres (éd.), Ethnicity and Resource Competition in Plural Societies, La Haye, Mouton Publishers, 1975, p. 9-21.

- JAuze J.M., «Tourisme et construction identitaire: exemple de l'île Maurice dans l'océan Indien", in P. Frustrier (dir), Les identités insulaires face au tourisme, La Rochesur-Yon, Siloë, 2007, p. 153-165.

- LaVABre M-C., «De la notion de mémoire à la production des mémoires collectives », in D. CEFAï (dir.), Cultures politiques, Paris, Presses universitaires de France, 2001, p. 233-252.

- Moses F., Mythe, Mémoire, Histoire. Les usages du passé, Paris, Flammarion, 1981.

- Nora P., Les lieux de mémoire, Paris, Gallimard, 1984.

- Paratian R., La république de l'île Maurice : dans le sillage de la délocalisation, Paris, L'Harmattan, 1994.

- Poirier C., «Mémoire collective, identité et politique. La société québécoise et sa relation problématique avec le passé», in I. Molinaro et C. Rolfe (éd.), Focus on Quebec 2. Further 
Essays on Quebecois Society and Culture, Édimbourg et Leceister, Le Greff, 2000, p. 73-87.

- Prabhu A., "Representation in Mauritian Politics : Who Speaks for the African Pasts ?» International Journal of Francophone Studies, vol. 8(2), 2005, p. 183-197.

- Ricceur P., La mémoire, l'histoire, l'oubli, Paris, Seuil, 2000.

- Ruiz Torres P., «Les usages politiques de l'histoire en Espagne. Formes, limites et contradictions", in F. HARTOG, J. Revel (dir.), Les usages politiques du passé, Paris, Éditions de l'École des hautes études en sciences sociales, 2001, p. 129-156.

- Schermerhorn R.A., Comparative Ethnic Relations, A Framework for Theory and Research, New York, Random House, 1968.
- Teelock V., «Historic Preservation : Whose History", in I. Asgarally (dir.), Colloque international Education et culture à l'aube du troisième millénaire, Floréal (Maurice), le Printemps, 1999, p. 73-81.

- Teelock V., «Breaking the Wall of Silence : Slavery in Mauritian Historiography », Radical History Review, n 91, 2005, p. 104-109.

- Todorov T., Les abus de la mémoire, Paris, Arléa, 1995.

- Vaughan M., Creating the Creole Island: Slavery in Eighteenth-Century Mauritius, Durham (NC), Duke University Press, 2005.

- Wieviorka M., Le racisme, une introduction, Paris, La Découverte, 1998. 